\title{
Towards a new Optimization Meta-Heuristic Inspired from Some Core Islamic Principles
}

\author{
Fateh Boutekkouk
}

\begin{abstract}
In this paper we trace the big lines of a new optimization meta-heuristic which is called Islamic Optimization Meta-Heuristic (IOMH) inspired from some core Islamic principles and especially from the cultural/social Islamic system perspectives. Our proposed meta-heuristic assumes that the society is composed of a certain number of clusters and each cluster is formed of a set of individuals. An individual inside a cluster can be strong Muslim, weak Muslim or non-Muslim. The idea of our optimization meta-heuristic is based mainly on the brotherhood in Allah and migration principles. Following the brotherhood principle the weak individuals in the cluster consolidate their fitness from strong individuals that translates to an intensified search around better solutions. However, the migration between clusters helps the search diversification around more better solutions.
\end{abstract}

Index Terms - Brotherhood in Allah, Islam, Migration, Optimization meta-heuristic.

\section{INTRODUCTION}

In contrast to exact mathematical methods in the field of combinatorial optimization, optimization meta-heuristics $(\mathrm{OMH})$ are general approximate approaches that are used to seek for near global optimal solutions in a reasonable time. $\mathrm{OMH}$ can be classified in static $\mathrm{OMH}$ with a fixed objective (cost) function or/and constraints and dynamic $\mathrm{OMH}$ where the objective function and/or the constraints can change over time. The literature is very rich; however we can state that most of existing $\mathrm{OMH}$ are biology and nature inspired. Some well known examples of such OMH are genetic algorithms and their variants, simulated annealing, Particle Swarm Optimization (PSO), ant and bee colonies, firefly algorithm, Bat Algorithm, Bacteria Foraging Optimization (BFO), Lion Optimization Algorithm (LOA), etc. [1, 4, 5, 8]. Other OMH are human memory behavior inspired as taboo search. An exhaustive survey on $\mathrm{OMH}$ can be found in [2].

Recently, researchers are being interested in $\mathrm{OMH}$ which are inspired from social interactions and cooperative behavior between humans [10]. For instance a new algorithm based on the simulation of social behavior using the two concepts of society and civilization was proposed [7].

A novel population-based optimization algorithm inspired by human group searching behavior in finding an optimal solution called Seeker optimization Algorithm or SOA.

Fateh Boutekkouk is with the Research Laboratory on Computer Science's Complex Systems ReLaCS2, University of Oum El Bouaghi, 04000, Algeria
In SOA, each search agent is called a seeker. The population is divided into $\mathrm{K}$ subpopulations and seekers in each subpopulation constitute a neighborhood. The new positions of seeks are guided by the direction towards the coordinate axis and the step length [3].

Another work proposed to use the concept of human opinion dynamics or propagation of belief among individuals as a basis for his optimization algorithm [9].

In this short paper, we try to inspire from Islam some core cultural/social rules or principles to develop an optimization meta-heuristic that can be tuned to any real specific optimization problem.

The great Islam [6] offers a set of well defined rules and recommendations that if strongly applied guarantee the safety, the richness (both moral and material) and the happiness of humanity. We can classify these rules in three classes:

1.Rules to manage the relation between the Muslim and Allah.

2.Rules to manage the relation between the Muslim and himself.

3. Rules to manage the relationship between the Muslim and the others (Muslims and non Muslims).

Muslim differs from non-Muslim by the fact that:

1.Muslim has an ultimate objective that is paradise.

2.Muslim is principle-driven not benefit-driven.

3.Muslim does not live only for his self but he lives also for the others because he considers all Muslims are brothers in Allah (for Muslims) or brothers in humanity (for non-Muslims).

4.Muslim practices religion in his life including economic, social, cultural, political and scientific terms. Islam is not just theoretical aspects or restricted only in Mosques: Islam is the life.

Muslim always furnishes continuously efforts to enhance or optimize his state. But what does he optimize?

1.Optimize his relationship with Allah.

2.Optimize his relationship with himself (honest, transparent, modest, etc.).

3. Optimize his relationship with other persons those can be blood-related, neighborhood-related, friends, colleagues, and others belonging to the same society or foreign society (respect, love, help, recommend, visit, etc.)

The result of such optimization in this life is certainly the happiness without doubt; all persons on this earth are seeking for happiness. Most of humans believe that happiness can be reached via money, travels, women, work, high level of 
education, etc. The right happiness comes from the inside not from the outside of our souls. Happiness is a logical consequence of belief, love and the application of recommendations of Islam.

As an example, rich Muslim must give some percent of his money to poor persons (In Islam we call this Zakat). By this way rich Muslims purify their souls from stinginess and greediness and poor Muslims from hostility towards rich Muslims. Consequently, the relationship between Muslims will be consolidated and the society will be balanced and optimized.

A general Islamic rule is that strong Muslim must help weak Muslim. The strength is a generic term that covers strength in belief in Allah, strength in knowledge and science, strength in money, strength in health, strength in power, etc.

One of the greatest missions of Muslim is the fact that he recommends other persons (Muslims and non Muslims) and conducts them to the right way. In this context Muslim feels that he is responsible towards the whole humanity, so that he spends his time, money and even his soul to accomplish his great objective.

In this context, we are developing our OMH which is called Islamic $\mathrm{OMH}$ or IOMH that applies the principle of brotherhood in Allah and the principle of migration that in its vast sense means the passage or moving from a bad (evil or wrong) state to a new good (righteous) state.

This paper is organized as follows. In section 2, the proposed meta-heuristic is overviewed informally. In section 3, the formal (mathematical) definition of our algorithm is presented. Section 4 presents an illustrative example and finally, our work is summarized in the last section with some short term perspectives.

\section{OVERVIEW OF OUR PROPOSED META-HEURISTIC}

We will assume that Islamic society consists in a set of communicating/cooperating clusters and each cluster is composed of a set of Muslims and may be a set of non-Muslims (i.e. Peaceful coexistence).

Strong Muslims must have a great impact on weak Muslims in the same society. In our context, a strong Muslim is a person who applies Allah guidelines strictly. In contrast, a weak Muslim may diverge from Islam guidelines. But even a Muslim is weak; we cannot eliminate him from the society rather than, we have to consolidate him and help him to become strong.

Individuals inside a cluster can be classified in two categories:

Strong Muslim individuals (SMI): are individuals whose fitness is higher than a certain threshold namely Th.

Weak Muslim individuals (WMI): are individuals whose fitness is less than Th. Th can be for instance the fitness mean of all individuals or another value introduced by the user.

SMI have a great impact on WMI and try to enhance them (good persons are good examples).

We assume that inside a cluster, a SMI must take at least one WMI as a brother in Allah (of course he can take more than one brother and it will be very advantageous especially if there is a big gap between them) and each individual has a position in the search space and a fitness which reflects the objective function value.

When the brotherhood in Allah is achieved, the position of the weak brother will be updated in a gradual fashion towards the region of the strong brother in hope of fitness improvement. During this movement, the fitness of the weak brother can surpass that of the strong one and thus becomes a strong but can also decrease or diverge from the SMI search region.

When the fitness of the weak brother is equal or greater than the strong brother, we update the position of the strong brother based on the best solution information from other clusters.

In general, an individual can migrate from his cluster to another cluster for the following reasons:

1.Because all individuals of his cluster are weak and this weak individual has the intention to enhance himself, so he migrates to another cluster with strong individuals.

2.Because one or some strong individuals of a cluster want to enhance individuals of other clusters.

3.An individual who migrates to another cluster must return to his original cluster when he accomplishes his mission.

\section{MATHEMATICAL FORMULATION}

Formally, let $f: \mathbb{R}^{d} \rightarrow \mathbb{R}$ be the objective function which must be maximized. The function takes a candidate solution as argument in the form of a vector of real numbers ( $d$ is the dimension) and produces a real number as output which indicates the objective function value of the given candidate solution. Strong individuals are the best solutions for the given problem (i.e. their objective function values are the highest ones among the others)

As a first attempt, we will assume that each SMI has to take a WMI as a brother in Allah while keeping the gap between them as higher as possible, so the best SMI will take the weakest WMI as a brother in Allah, the second best SMI will take the second weakest and so on. In such away, some individuals can remain without brothers.

This situation occurs when the number of individuals inside a cluster is impair; that leads to individuals whose fitness is equal to the all individuals fitness mean. We call these special individuals neutral Muslim individuals or NMI. Let:

$\mathrm{K}$ be the number of clusters,

$\mathrm{M}$ be the number of individuals inside a cluster,

Th be the threshold,

In the total, we have $\mathrm{N}=\mathrm{K} * \mathrm{M}$ individuals. Initially for each individual, we generate his vector of position $\mathrm{P}$ in the search space of dimension $\mathrm{d}$ randomly then for each individual, we compute his objective function value.

In our proposed algorithm we will normalize the values of objective function using the feature scaling technique, so we bring all values into the range [0.1]. The formula used for normalization is:

$$
\mathrm{F}^{\prime}=(\mathrm{F}-\mathrm{Fmin}) /(\mathrm{Fmax}-\mathrm{Fmin})
$$

Each cluster will contain one of the $\mathrm{K}$ first ordered SMI, the remaining M-1 individuals are distributed randomly over the $\mathrm{K}$ 
clusters. Since we have no clear idea (due to the lack of statistical/mathematical studies on this topic) on the impact of the brotherhood in Allah on the moving and dynamics of Muslims, we propose to use simple but novel formulas to update the position of WMI, SMI and NMI.

The general idea is that very good solutions (SMI brothers) try to attract gradually weak solutions (WMI brothers) to their search regions (i.e. their positions in the search space) but at the same time and in order to avoid dropping in local optimum, we authorize weak solutions to diverge sometimes from the search regions of SMI. On the other side, SMI have the possibility to extend their search regions by updating their positions either when the fitness of the weak brother surpasses or equals the SMI fitness or after a certain time period (a certain number of iterations fixed by the user) during which we don't observe any significant improvement in the fitness of the WMI. In all cases, we have to memorize at every iteration, the WMI best position and its corresponding fitness.

NMI will move randomly.

1.Between SMI and WMI : once the brotherhood in Allah is achieved, both SMI and WMI positions will be updated as follows:

Where:

$$
\operatorname{Pw}(t+1)=\operatorname{Pw}(t)+d_{1} \cdot \alpha+d_{2} \cdot \beta
$$

$\mathrm{Pw}(\mathrm{t}+1)$ is the new position of the WMI in iteration $t+1$.

$\mathrm{Pw}(\mathrm{t})$ is the position of the WMI in iteration $\mathrm{t}$ (the old position).

$f(\mathrm{Pw}(\mathrm{t}))$ is the normalized objective function value for the WMI in iteration $\mathrm{t} .(f(\mathrm{Pw}(\mathrm{t})) \in[0,1])$.

$f(\operatorname{Ps}(\mathrm{t}))$ is the normalized objective function value for the SMI in iteration t. $(f(\operatorname{Ps}(\mathrm{t})) \in[0,1])$.

$\alpha \in[0,1]$ denotes the step of moving of the weak brother

$$
\alpha=f(\operatorname{Ps}(\mathrm{t}))-f(\operatorname{Pw}(\mathrm{t}))
$$

With $\alpha \in[0,1]$, the search space will be explored smoothly (to encourage the intensification) and this will help to don't miss good solutions.

$\beta \in[0,1]$ denotes the factor of regression towards weakness (i.e. it help the WMI to diverge from SMI search region). $\beta$ is generated randomly. The role of this factor is to avoid falling in local optimum.

$\mathrm{d}_{1}$ indicates the direction of the WMI towards SMI:

Two cases can appear:

if the position of WMI < the position of SMI then

$\mathrm{d}_{1}=1$

if the position of WMI > the position of SMI then

$\mathrm{d}_{1}=-1$

$\mathrm{d}_{2}$ indicates the reverse direction of the WMI towards SMI:

Two cases can appear:

if the position of WMI < the position of SMI then

$\mathrm{d}_{2}=-1$

if the position of WMI > the position of SMI then

$\mathrm{d}_{2}=1$

The idea behind this formula is that more the gap between the SMI and the WMI is higher, more the step of moving $\alpha$ towards the SMI region is higher which reflects a high attraction and vice versa.

For SMI, his update formula is:

$$
\begin{gathered}
\operatorname{Ps}(\mathrm{t}+1)=\operatorname{Ps}(\mathrm{t})+\mathrm{d} . \delta . \alpha \\
\alpha=f(\operatorname{PBs}(\mathrm{t}))-f(\operatorname{Ps}(\mathrm{t})) \quad \text { with } \alpha \in[0,1]
\end{gathered}
$$

$f(\operatorname{PBs}(\mathrm{t})$ is the normalized objective function value of the best solution among all clusters.

$\mathrm{d}$ indicates the direction and may be 1 or -1 . It is calculated similarly to $\mathrm{d}_{1}$

If SMI is the best ever, then its formula becomes:

$$
\operatorname{Ps}(\mathrm{t}+1)=\operatorname{Ps}(\mathrm{t})+\mathrm{d} . \delta . \alpha
$$

$\mathrm{d}$ is a random number indicating the direction and may be 1 or -1 .

$\alpha$ is a random number $\epsilon[0,1]$.

In order to encourage the diversification, we introduce a number $\delta>1$, we call it the diversification factor, used to larger the moving step (strong individuals are capable of jumping longer than weak ones). The exact value of $\delta$ depends on the scale of the individual positions and it is introduced by the user.

We remark that the step of moving of SMI in the search space is controlled by the best solution ever among all the clusters and multiplied by $\delta$. Such big steps will compromise the small steps used with WMI. Over time, SMI can become WMI or NMI.

For NMI, his update formula is:

$$
\operatorname{Pn}(\mathrm{t}+1)=\operatorname{Pn}(\mathrm{t})+\mathrm{d} . \delta \alpha_{\mathrm{n}}
$$

$\alpha_{\mathrm{n}} \in[0,1]$ is generated randomly.

The NMI is better than WMI (i.e. his jump is longer than the WMI), for this reason, we choose $\delta>1$ but its value is less than the diversification factor for SMI.

This process is repeated until a certain termination criterion is satisfied. The termination criterion may be for instance the maximum number of iterations or a certain threshold on the objective function value.

In our proposed algorithm, we interpreted the migration principle as information sharing between SMI inside clusters: that means each SMI inside a cluster has knowledge about the best solution of each cluster.

The pseudo code of our algorithm looks like:

1.Generate the positions of $\mathrm{N}$ individuals randomly in the search space of dimension $\mathrm{d}$.

2.For each individual, compute his objective function and normalize it using eq. 1

3.Divide $\mathrm{N}$ individuals into $\mathrm{K}$ clusters of $\mathrm{M}$ individuals. Each cluster will contain one of the $\mathrm{K}$ first ordered SMI, the remaining M-1 individuals are distributed randomly over the $\mathrm{K}$ clusters.

4.Global_best $\leftarrow$ the first ordered SMI;

5.While stop criterion is not satisfied do

6.For each cluster do

7. Make the best SMI, brother in Allah with the weakest one, the second best with the second weakest, and so on.

8.If there is a NMI then

9.Update his position using eq.7, evaluate $f_{\mathrm{NMI}}$ and normalize it.

10.for each couple of brothers in Allah do 
11.Update WMI position using eq.2, evaluate $f_{\mathrm{WMI}}$ and normalize it.

12.Memorize WMI_best;

13. If WMI_best $\geq f_{\text {SMI }}$ then

14.Update SMI position using eq. 4 or eq.6, evaluate $f_{\text {SMI }}$ and normalize it.

15.Else

16.If delay_expired then

17.Update SMI position using eq.4 or eq.6, evaluate $f_{\text {SMI }}$ and normalize it

18.Else goto 11

19.End if

20.End if

21.WMI_current $\leftarrow$ WMI_best

22.End for

23.Calculate Best_cluster;

24.End for

25.Calculate Global_best among Best_clusters

26.End while

Our proposed algorithm is sensible to many parameters:

1. $\alpha$

2. $\beta$,

3. The diversification factor $\delta$,

4. $\alpha_{\mathrm{n}}$

5. The delay of expiration,

6 . The termination criterion.

Although the algorithm is presented in a sequential fashion, we can easily parallelize it (i.e. each cluster can be seen as a thread) and consequently we can gain much time.

\section{ILLUSTRATIVE EXAMPLE}

In order to study the behavior of our proposed algorithm, we start by a simple example that tries to maximize the function:

$\sum_{i} x_{i}^{2}$ with $\mathrm{i}=1,2,3$ and $\mathrm{x}_{\mathrm{i}} \in[0,1]$

We have done some experimentations relying on changing the parameter $\delta_{\mathrm{n}}\left(\delta\right.$ for NMI) and $\delta_{\mathrm{s}}(\delta$ for SMI). We assume that $\mathrm{N}=30, \mathrm{~K}=3$, and $\mathrm{M}=10$. The expired delay $=10$. Figures 1,2 , and 3 show the progression of the research process over 1000 iterations when $\delta_{\mathrm{n}}=\delta_{\mathrm{s}}=1 ; \delta_{\mathrm{n}}=2$ and $\delta_{\mathrm{s}}=4 ; \delta_{\mathrm{n}}=5$ and $\delta_{\mathrm{s}}=10$ respectively. According to our first observations, we can see that in the first case, the function progresses smoothly and the behavior looks like a gradient. The function risks of a premature convergence. However, the function in cases two and three does not progress smoothly because the research is more diversified thus the obtained results match well our first intuition.

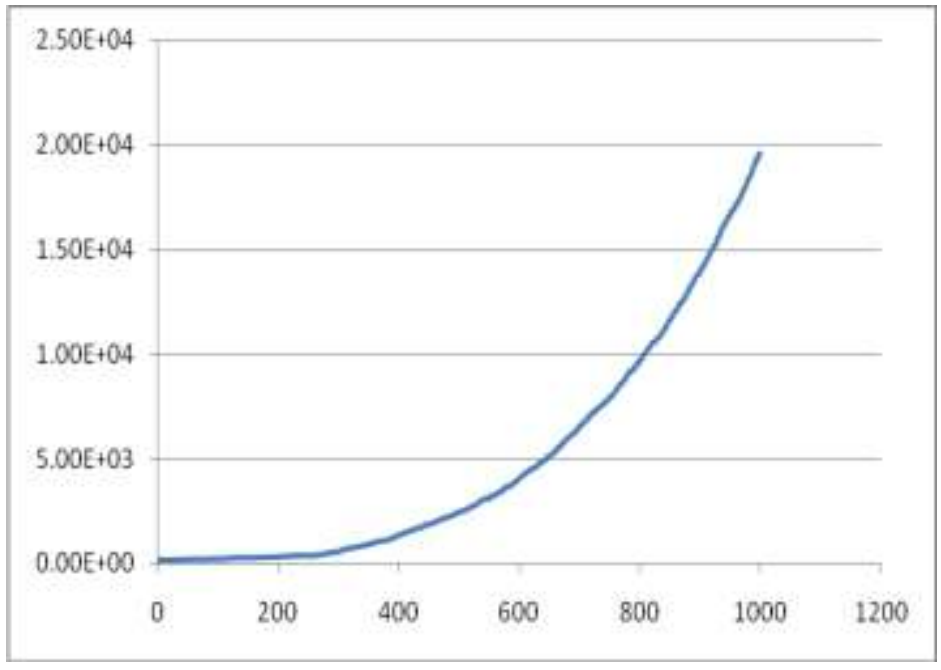

Fig. 1. Result for $\delta_{n}=1 ; \delta_{s}=1$.

\section{CONCLUSION}

This work deals with the development of a new optimization meta-heuristic inspired from some core Islam perspectives and especially the cultural/social Islamic system. The two principles are the brotherhood in Allah and the migration. As a first attempt, we have proposed some formulas to update the individuals' positions. As a perspective, we plan to test our algorithm on many benchmarks, fix the best values of $\beta, \delta, \alpha_{n}$, possibly modify or enhance the formula calculating $\alpha$ and make concrete comparisons with other well known meta-heuristics.

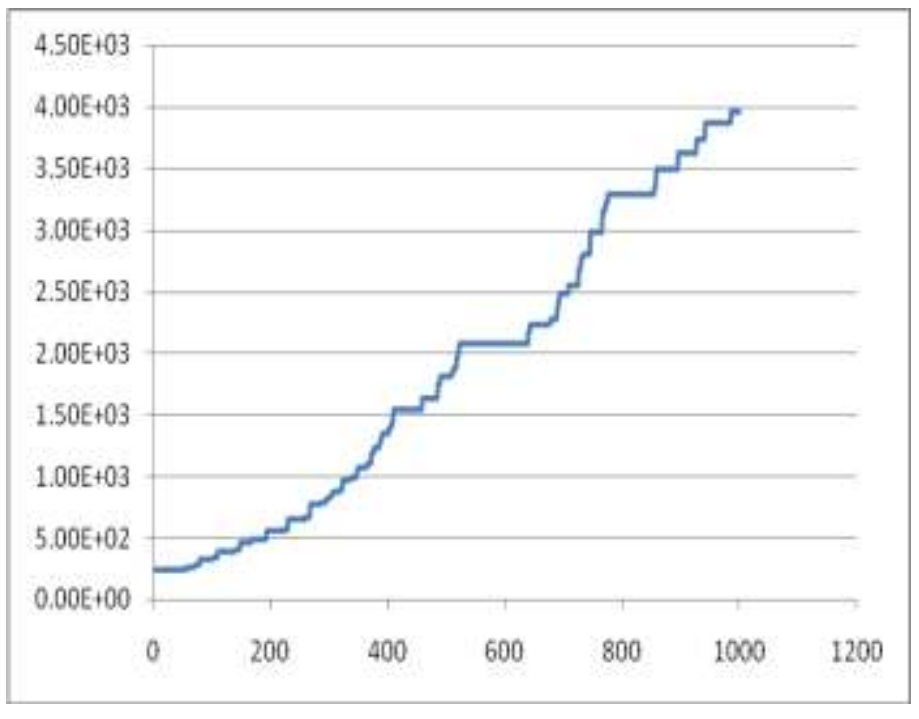

Fig. 2. Result for $\delta_{\mathrm{n}}=2 ; \delta_{\mathrm{s}}=4$. 


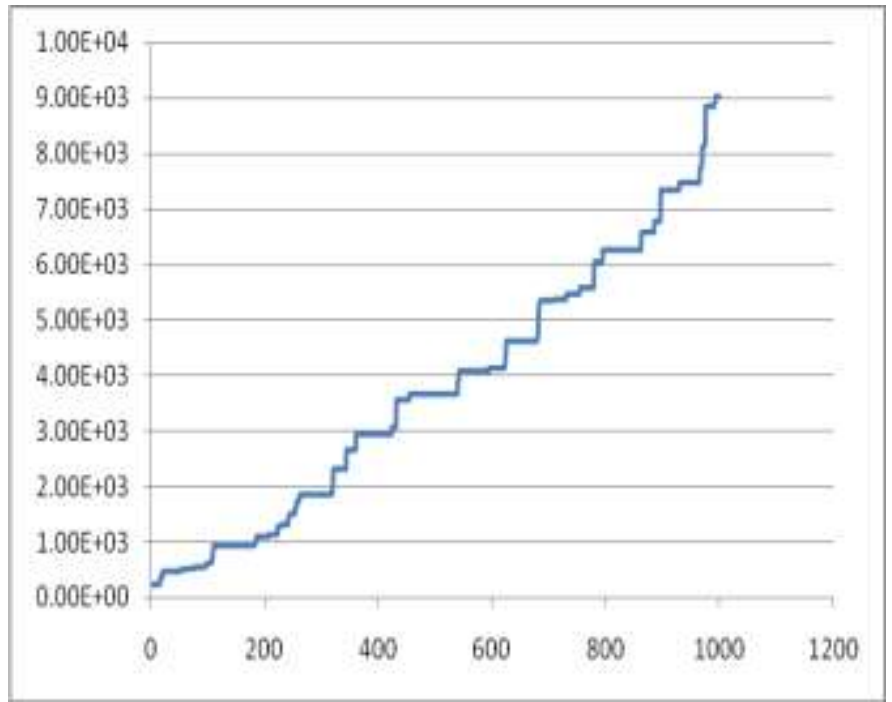

Fig. 3. Result for $\delta_{\mathrm{n}}=5 ; \delta_{\mathrm{s}}=10$.

\section{REFERENCES}

[1] S. Binitha, S.S. Sathya, "A Survey of Bio inspired Optimization Algorithms" in International Journal of Soft Computing and Engineering (IJSCE), 2012, Volume-2, Issue-2.

[2] I. Boussaï, J. Lepagnot, P. Siarry, "A survey on optimization metaheuristics" in Information Sciences 237, 2013, 82-117. https://doi.org/10.1016/j.ins.2013.02.041

[3] C. Dai, Y. Zhu, W. Chen, "Seeker optimization algorithm". In Computational intelligence and security. Lecture Notes in Computer Science. Berlin Heidelberg: Springer, Vol. 4456, 2007, 167-176. https://doi.org/10.1007/978-3-540-74377-4_18

[4] F. Iztok, Y. Xin-She, B. Janez, "A comprehensive review of firefly algorithms" a survey paper Swarm and Evolutionary Computation 13 2013, 34-46. https://doi.org/10.1016/j.swevo.2013.06.001

[5] A. R. Jordehi, J. Jasni, "Particle swarm optimisation for discrete optimisation problems: A review". Artificial Intelligence Review, 43, 2013, 243-258. https://doi.org/10.1007/s10462-012-9373-8

[6] Z. Khan, M. Farooq, A. Ullah, "Optimization of Consumption in Divine Context: (Basic Principles and Extension)". Al-Idah 24 June, 2012.

[7] K.M Liew, "Society and civilization: An optimization algorithm based on the simulation of social behavior" in IEEE Trans. Evol. Comput. 7 2003, 386-396.

[8] Y. Maziar, J. Fariborz, "Lion Optimization Algorithm (LOA):A nature-inspired metaheuristic algorithm" in Journal of Computational Designand Engineering 3, 2016, 24-36.

[9] K. Rishemjit, K. Ritesh, P. B. Amol, K. Pawan, "Human opinion dynamics: An inspiration to solve complex optimization problems" scientific report, 2013.

[10] A. Yuji, "How to measure social interactions via group selection? Cultural group selection, coevolutionary processes, and large-scale cooperation: a comment." in Journal of Economic Behavior \& Organization, Vol. 53, 2004, 41-47.

https://doi.org/10.1016/S0167-2681(03)00101-X

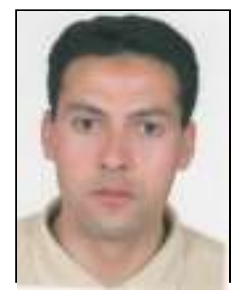

Fateh Boutekkouk is a senior lecturer at the university of Oum El Bouaghi, Algeria. He obtained his doctorate degree in 2010 from the University of Constantine, Algeria. His main research includes: Real Time embedded systems, systems on a chip, and network on a chip design, intelligent distributed embedded systems design, software engineering and formal techniques. 